\title{
Desempenho de frangos de corte criados na região oeste do Paraná em relação à linhagem e sistemas de produção
}

\author{
Performance of broiler chickens reared in the western region of Paraná in \\ relation to genetic line and production systems
}

\begin{abstract}
Lucas de Almeida Reati (D) ${ }^{*}$, Gabriela Rocha Santos (D) ${ }^{1}$, Izabela Camilotti Dorneles (D)2 , Eduardo Herrera Dias (D)², Taniara Suelen Mezalira (D) ${ }^{2}$, Maurício Fanin (D) ${ }^{2}$, Alysson Polzonoff Silveira (D) 2,3, Andréia Assunção Soares (D) 1,2, Luciana Kazue Otutumi (D) ${ }^{1,2}$

${ }^{1}$ Curso de Medicina Veterinária, Universidade Paranaense (UNIPAR), Umuarama, PR, Brasil

2 Programa de Pós-Graduação em Ciência Animal com Ênfase em Produtos Bioativos, Universidade Paranaense (UNIPAR), Umuarama, PR, Brasil

${ }^{3}$ Nutrição Animal, Oceana Minerals, Jundiaí, SP, Brasil
\end{abstract}

\section{Resumo}

O objetivo desse trabalho foi comparar o desempenho zootécnico e o custo de produção de diferentes linhagens de frangos de corte (Cobb Slow, Cobb Fast e Hubbard), sistemas de ventilação (positiva e negativa) e tipos de aviário (lona amarela/convencional, blue house e dark house). Foi realizado um levantamento de dados de desempenho e custo de duas granjas avícolas localizadas na região oeste do estado do Paraná, no período de 25 de setembro de 2012 a 25 de setembro de 2017. Verificou-se que as linhagens Hubbard e Cobb Slow apresentaram melhores resultados nas variáveis índice de eficiência produtiva (IEP) $(356,3$ vs. 334,6) e peso médio final $(3,014 \mathrm{~kg}$ vs. $2,902 \mathrm{~kg})$. A linhagem Hubbard também mostrou significativamente maior ganho médio diário de peso (GMDP) em relação às duas outras linhagens, com uma média de 64,4 g. Em relação ao sistema de ventilação, verificou-se que a ventilação negativa é mais eficiente do que a positiva quando avaliado o GMDP, e que os sistemas dark e blue house mostraram um desempenho superior ao sistema convencional (lona amarela). Conclui-se que animais da linhagem Hubbard e Cobb Slow apresentam melhores

* Autor correspondente: lucasdealmeidareati@gmail.com 
resultados de desempenho zootécnico (IEP, peso médio) do que animais da linhagem Cobb Fast. Em relação ao sistema de ventilação (positiva e negativa) e tipos de aviário (dark house, blue house e convencional), melhores resultados de desempenho zootécnico são observados para sistemas que propiciam melhor ambiência, ou seja, ventilação negativa e com controle de luminosidade (dark e blue house). Por outro lado, em termos de custos, não houve diferenças nos parâmetros avaliados.

Palavras-chave: Avicultura. Dark house. Linhagem Cobb. Linhagem Hubbard.

\section{Abstract}

The objective of this work was to compare the zootechnical performance and the production cost of different broiler lines (Cobb Slow, Cobb Fast and Hubbard), ventilation systems (positive and negative), and canvas types (yellow/conventional), blue house, and dark house). A performance and cost data survey was carried out on two poultry farms located in the western region of the state of Paraná from September 25, 2012 to September 25,2017 . It was verified that the genetic line Hubbard and Cobb Slow showed better results in the variables productive efficiency index (IEP) $(356,3$ vs. 334,6) and final weight $(3.014 \mathrm{~kg}$ vs. $2.902 \mathrm{~kg})$. The genetic line Hubbard also showed significantly greater average daily gain $(A D G)$ in relation to the two others lineages, with an average of $64,4 \mathrm{~g}$. Regarding the ventilation system, it was found that negative ventilation is more efficient than positive when ADG was evaluated, and the dark and blue house systems showed a better performance than the conventional one (yellow canvas). It is concluded that animals of the Hubbard and Cobb Slow lineage have better zootechnical performance results (IEP, average weight) than animals of the Cobb Fast lineage. In relation to the ventilation system (positive and negative) and aviary types (dark house, blue house and conventional), better results of zootechnical performance are observed for systems that provide better ambience, that is, negative ventilation and with light control (dark and blue house). On the other hand, in terms of costs, there were no differences in the parameters evaluated.

Keywords: Poultry farming. Dark house. Cobb broiler. Hubbard broiler.

\section{Introdução}

No ano de 2019, o Brasil produziu 13,24 milhões de toneladas de carne de frango, o que conferiu ao país o terceiro lugar no ranking mundial, sendo os estados do Paraná, Santa Catarina e Rio Grande do Sul os maiores produtores. Já na exportação, o país ocupa o primeiro lugar no ranking mundial, tendo exportado 4,21 milhões de toneladas no mesmo ano (ABPA, 2020). Parte dessa evolução ocorreu devido às pesquisas realizadas na área de melhoramento genético, nutrição, ambiência, sanidade e abate (Machado et al., 2014).

O melhoramento genético dos frangos tem introduzido no mercado linhagens mais modernas de alto rendimento produtivo (Arruda, 2013), de tal forma que o Brasil conta com diversas linhagens de frangos de corte como Hubbard, Isa, Cobb, Ross, Arbor Acres e Shaver (Lopes, 2011). Segundo o mesmo autor, uma boa conversão alimentar, rápido crescimento, uniformidade, empenamento precoce, peito largo, pernas curtas, boa pigmentação da pele e resistência a doenças são algumas características desejáveis para a produção de carne.

Além disso, a evolução científica e tecnológica na qual o Brasil está inserido e as tendências mundiais para um desenvolvimento sustentável fazem com que os profissionais que atuam na área de ambiência tenham visões direcionadas para novos horizontes (Silva e Vieira, 2010). Segundo Abreu e Abreu (2011), nas últimas décadas a avicultura de corte tem investido em inovações tecnológicas, o que permitiu a adoção de novos sistemas de criação de frangos de corte, como o sistema dark house, onde as instalações permitem melhor controle interno da iluminação e das condições térmicas, como temperatura, umidade e velocidade do vento. Esse sistema conta com exaustores em pressão negativa, nebulizadores, light-trap para controle da luz natural e também fazem o uso de dimmer para o controle da intensidade da luz. Para garantir bons resultados nesse sistema e consequente melhor desempenho das aves, é necessário que o aviário seja bem isolado e vedado, para que não haja influência do ambiente externo nas condições de ambiência no interior do aviário. 
Os aviários do tipo blue house também são muito usados na criação de frangos de corte no Brasil (Carvalho et al., 2011). Assim como os modelos dark house, também são sistemas climatizados de produção (Souza et al., 2017), porém eles têm como característica principal o uso das cortinas na cor azul para controlar a luminosidade e, consequentemente, a atividade das aves (Carvalho et al., 2011). O uso da lona azul tem se mostrado como um ponto positivo no crescimento desses animais, uma vez que ela permite, por meio do controle da luminosidade, que as aves se agitem menos e, assim, ganhem mais peso. Além disso, o sistema blue house também permite que o produtor tenha menor custo com mão de obra, fazendo-se necessário apenas um funcionário, desde que o mesmo tenha domínio do equipamento responsável pela sistematização do galpão (Avicultura Industrial, 2017). A principal finalidade da variação na cor da lona utilizada nesses galpões é o controle da intensidade luminosa, uma vez que interferem na quantidade de luz penetrada e reduzem a movimentação dos animais dentro do galpão, ressaltando, mais uma vez, a vantagem que aviários no estilo blue e dark house apresentam sobre os demais modelos (Revista Rural, 2009).

O sistema semiclimatizado, segundo Abreu e Abreu (2011), possui comedouro tubular, bebedouro pendular ou nipple; não possui sistema de controle artificial da temperatura e o condicionamento térmico é natural, sendo que para este sistema os galpões possuem ventiladores em pressão positiva, podendo ou não possuir forro, e com cortina de ráfia amarela, azul ou branca. Os mesmos autores ainda definem como sistema climatizado aquele que apresenta maior controle das condições térmicas ambientais quando comparado ao sistema semiclimatizado: ventiladores em pressão positiva ou exaustores em pressão negativa com sistema de resfriamento por nebulização e/ou pad cooling, podendo ter ou não forro ou defletores com cortina de ráfia amarela, azul ou branca.

O estresse térmico é um dos principais fatores de redução no desempenho produtivo de animais. Uma vez que as aves precisam fazer uso de meios que auxiliem sua termorregulação, sua capacidade produtiva é reduzida (Souza et al., 2017). Dessa forma, o objetivo do presente trabalho foi comparar o desempenho zootécnico e o custo de produção de três linhagens de frangos de corte (Cobb Slow, Cobb Fast e Hubbard), dois sistemas de ventilação (positiva e negativa) e três tipos de aviário (convencional/lona amarela, blue house e dark house).

\section{Material e métodos}

Foi realizado um levantamento de dados de duas granjas avícolas da região oeste do estado do Paraná, no período de 25 de setembro de 2012 a 25 de setembro de 2017. Uma das granjas possuía dois aviários de frangos de corte (aviário convencional com sistema climatizado, lona amarela e ventilação positiva), com 68 metros de comprimento por 12 de largura $\left(816 \mathrm{~m}^{2}\right)$, capacidade de alojamento de 8500 aves cada, duas linhas de comedouro automático, quatro linhas de bebedouro tipo nipple e doze ventiladores distribuídos no interior do galpão.

A outra granja possuía igualmente dois aviários de frangos de corte, sendo um com 130 metros de comprimento por 12 de largura $\left(1560 \mathrm{~m}^{2}\right)$ e capacidade de alojamento de 20.000 aves, sistema de ventilação negativa com oito exaustores, nebulizadores, painel evaporativo (tijolo cerâmico), lona azul (aviário tipo blue house). O outro aviário era do tipo dark house, com 130 metros de comprimento por 14 metros de largura $\left(1820 \mathrm{~m}^{2}\right)$, capacidade de alojamento de 25.000 aves, dez exaustores, nebulizadores e painel evaporativo (celulose).

Os seguintes dados de desempenho foram tabulados: idade de abate (dias), mortalidade (\%), conversão alimentar $(\mathrm{kg} / \mathrm{kg})$, consumo médio de ração $(\mathrm{kg})$, índice de eficiência produtiva (IEP), ganho médio diário de peso (GMDP) ( $g$ ), peso médio $(\mathrm{kg})$ no intervalo entre os dias 45-49 de idade, valor por cabeça (reais), custo por cabeça (reais) e custo por quilo (reais).

O IEP, muito utilizado pelas empresas para remunerar seus integrados, é avaliado por meio dos parâmetros GMDP $(\mathrm{kg})$, viabilidade (\%) e conversão alimentar $(\mathrm{kg} / \mathrm{kg})$. O valor do IEP é 
calculado por uma divisão onde o numerador é obtido pela multiplicação entre GMDP e viabilidade, e o denominador é a conversão alimentar. Já a conversão alimentar é calculada pelo consumo total de ração, dividido pelo peso médio das aves. Os custos por cabeça e por quilo são calculados respectivamente levando-se em consideração a quantidade de animais entregue e a quantidade em quilos entregue na plataforma de abate.

Os dados foram tabulados no programa Excel versão 2016 e analisados utilizando-se o programa estatístico IBM SPSS v.21.0. Os dados foram primeiramente examinados em relação à normalidade (Shapiro-Wilk) e homogeneidade de variância (teste de Levene). Os dados de desempenho e de custos que não apresentaram distribuição normal e homogeneidade de variância foram analisados pelo teste KruskalWallis; para os que apresentaram, optou-se pela análise de variância seguida de pós-teste de Tukey HSD. Esses dados foram analisados em função da linhagem (Cobb Slow, Cobb Fast e Hubbard) e tipo de aviário (convencional/lona amarela, blue house e dark house). Para a comparação de dois grupos (ventilação positiva e negativa), utilizou-se o teste $\mathrm{U}$ de Mann-Whitney de amostras independentes. Para todos os testes foi considerado nível de significância de $5 \%$.

\section{Resultados e discussão}

Em relação à linhagem (Cobb Slow, Cobb Fast e Hubbard), verificou-se um maior IEP para os animais da linhagem Hubbard quando comparados com os da linhagem Cobb Fast, não havendo diferenças em relação à linhagem Cobb Slow (Tabela 1). No entanto a linhagem Hubbard apresentou significativamente maior GMDP em relação às duas outras linhagens. Resultado similar foi verificado para o peso médio final, com maior peso $(p<0,05)$ para os animais da linhagem Hubbard quando comparados com os da linhagem Cobb Fast, não havendo diferença para a linhagem Cobb Slow.

As demais variáveis estudadas, idade média de abate, mortalidade, conversão alimentar, consumo médio de ração, custo por cabeça e por quilo, não foram influenciadas pela linhagem (Tabela 1).

Tabela 1 - Média \pm erro padrão das variáveis de desempenho e custo de frangos de corte em função das linhagens Cobb Slow, Cobb Fast e Hubbard

\begin{tabular}{lcccc}
\hline \multirow{2}{*}{ Variáveis } & \multicolumn{2}{c}{ Linhagem } & Valor de p \\
\cline { 2 - 4 } & Cobb Slow & Cobb Fast & Hubbard & 0,475 \\
\hline Data de abate (dias) & $47,9 \pm 0,3$ & $47,1 \pm 0,3$ & $46,8 \pm 0,3$ & 0,679 \\
Mortalidade $(\%)^{1 *}$ & $4,0 \pm 0,3$ & $3,5 \pm 0,3$ & $4,0 \pm 0,7$ & 0,069 \\
Conversão alimentar (kg/kg) & $1,769 \pm 0,0$ & $1,785 \pm 0,01$ & $1,738 \pm 0,02$ & 0,170 \\
Consumo médio (kg) & $5,131 \pm 0,0$ & $5,049 \pm 0,0$ & $5,234 \pm 0,0$ & 0,002 \\
Índice de eficiência produtiva (IEP) & $334,6 \pm 3,3^{\mathrm{ab}}$ & $325,5 \pm 4,0^{\mathrm{b}}$ & $356,3 \pm 7,5^{\mathrm{a}}$ & 0,000 \\
Ganho médio diário de peso (g) & $61,5 \pm 0,4^{\mathrm{b}}$ & $60,0 \pm 0,5^{\mathrm{c}}$ & $64,4 \pm 0,6^{\mathrm{a}}$ & 0,005 \\
Peso médio (kg) & $2,902 \pm 0,0^{\mathrm{ab}}$ & $2,833 \pm 0,0^{\mathrm{b}}$ & $3,014 \pm 0,0^{\mathrm{a}}$ & 0,411 \\
Valor por cabeça (reais) & $0,58 \pm 0,0$ & $0,54 \pm 0,0$ & $0,56 \pm 0,0$ & 0,888 \\
Custo por cabeça (reais) & $5,9 \pm 0,2$ & $5,9 \pm 0,2$ & $5,7 \pm 0,2$ & 0,221 \\
Custo por kg (reais) & $2,0 \pm 0,0$ & $2,0 \pm 0,0$ & $1,9 \pm 0,0$ & 0
\end{tabular}

Nota: ${ }^{1}$ Não significativo pelo teste Kruskal-Wallis. ${ }^{2}$ Não significativo pela análise de variância. * Mortalidade do período de criação (média de 47 dias). Médias seguidas de letras diferentes na linha diferem pelo teste de Tukey HSD. 
Garcia Neto e Campos (2004) verificaram menor ganho de peso para as aves da linhagem Cobb quando comparadas às aves das linhagens Ross e Hubbard aos 39 dias de idade. Resultados semelhantes foram encontrados por Moreira et al. (2003) em estudo avaliando o desempenho em frangos das linhagens Ross 308, Cobb 500 e Hubbard aos 42 dias de idade. Os autores verificaram maior ganho de peso para a linhagem Hubbard (2464 g) e Ross (2433 g) quando comparadas com a Cobb 500 (2311 g). Por outro lado, resultados obtidos por Api et al. (2017) não demonstraram diferenças significativas no ganho de peso para as linhagens Ross, Cobb e Hubbard aos 45 dias. Embora os autores tenham observado menor peso médio nas primeiras semanas para a linhagem Hubbard, no final do período avaliado (45 dias) não houve diferenças significativas entre elas, evidenciando que as diferenças nas curvas de crescimento resultam de características genéticas.

Pereira et al. (2019) compararam o desempenho zootécnico de três linhagens diferentes e verificaram que a linhagem Hubbard apresentou valores semelhantes a Cobb para peso médio inicial, porém, quando analisado o peso médio e o ganho médio de peso, as aves Hubbard obtiveram melhor desempenho em relação às linhagens Cobb e Ross. A conversão alimentar também foi comparada, onde os animais Hubbard apresentaram valores inferiores aos demais.

De acordo com a Associação Paranaense de Avicultura (APAVI, 2009), os índices zootécnicos podem ser definidos como o padrão de desenvolvimento ou produção da granja, onde são considerados coeficientes como conversão alimentar, GMDP, idade de abate, IEP, taxa de mortalidade, peso de abate, viabilidade e ração consumida, servindo como guias de criação, referência e metas a serem atingidas.

O IEP é muito utilizado pelas empresas para remunerar seus integrados e o seu valor vem aumentando significativamente nos últimos anos devido às melhorias nos mais diferentes segmentos da produção, como nutrição, genética, sanidade, manejo, ambiência, entre outros (Moro et al., 2005). Dados de uma cooperativa produtora de frango de corte apontaram números expressivos e inéditos IEP com média de 366 pontos no fechamento do mês de fevereiro de 2018, com conversão alimentar média de 1,69 kg/kg (Avicultura Industrial, 2018).

Em relação aos tipos de ventilação, verificouse diferença $(p<0,05)$ para as variáveis conversão alimentar, IEP, GMDP, peso médio e valor por cabeça, com os animais criados em sistema de ventilação negativa apresentando melhores resultados de desempenho para as variáveis citadas (Tabela 2 ).

Tabela 2 - Média \pm erro padrão das variáveis de desempenho e custo de frangos de corte em função da ventilação positiva e negativa

\begin{tabular}{|c|c|c|c|}
\hline \multirow{2}{*}{ Variáveis } & \multicolumn{2}{|c|}{ Ventilação } & \multirow{2}{*}{ Valor de $p$} \\
\hline & Positiva & Negativa & \\
\hline Data de abate (dias) ${ }^{1}$ & $46,9 \pm 0,2$ & $47,3 \pm 0,2$ & 0,241 \\
\hline Mortalidade $(\%)^{1 \star}$ & $3,7 \pm 0,3$ & $3,9 \pm 0,3$ & 0,291 \\
\hline Conversão alimentar (kg/kg) & $1,799 \pm 0,0^{\mathrm{a}}$ & $1,744 \pm 0,0^{b}$ & 0,000 \\
\hline Consumo médio $(\mathrm{kg})$ & $5,113 \pm 0,0$ & $5,096 \pm 0,0$ & 0,906 \\
\hline Índice de eficiência produtiva (IEP) & $325,7 \pm 3,8^{b}$ & $340,6 \pm 3,0^{a}$ & 0,001 \\
\hline Ganho médio diário de peso (g) & $60,5 \pm 0,5^{b}$ & $61,7 \pm 0,3 a$ & 0,032 \\
\hline Peso médio $(\mathrm{kg})$ & $2,843 \pm 0,0^{b}$ & $2,923 \pm 0,0^{a}$ & 0,013 \\
\hline Valor por cabeça (reais) & $0,47 \pm 0,0^{b}$ & $0,65 \pm 0,0^{a}$ & 0,000 \\
\hline Custo por cabeça (reais) $^{1}$ & $5,7 \pm 0,1$ & $5,9 \pm 0,1$ & 0,266 \\
\hline Custo por kg (reais) & $2,0 \pm 0,0$ & $2,0 \pm 0,0$ & 0,782 \\
\hline
\end{tabular}

Nota: ${ }^{1}$ Não houve diferença pelo Teste U de Mann-Whitney de amostras independentes.* Mortalidade do período de criação (média de 47 dias). Médias seguidas de letras diferentes na linha diferem pelo teste-t para igualdade de médias. 
O sistema de ventilação e de resfriamento utilizado em instalações para frangos de corte desempenha um papel importante no controle ambiental, possibilitando que a ave expresse seu potencial máximo de produção (Curi et al., 2014).

A ventilação com o uso de ventiladores, também chamada de ventilação positiva, associada à nebulização e manejo de cortinas, é um sistema que visa manter as aves em um ambiente térmico ideal. No entanto, os aviários equipados com exaustores (ventilação negativa) associados a nebulizadores ou a placas evaporativas também visam criar as aves dentro de condições térmicas ideais para o desenvolvimento e melhor produtividade (Rovaris et al., 2014). O sistema de ventilação negativa, por permitir melhor controle das condições de ambiência, justifica os melhores resultados de desempenho encontrados no presente trabalho.

Rovaris et al. (2012), analisando dados de uma empresa situada em uma região considerada quente, com média de temperatura de $34^{\circ} \mathrm{C}$ e $80 \%$ de umidade, obtiveram melhores resultados de GMDP e conversão alimentar nos frangos de corte criados em sistema dark house (ventilação negativa) quando comparados àqueles de aviários convencionais. De acordo com Rovaris (2013),
- sistema convencional de criação de frangos de corte consiste em aviários com lonas azuis ou amarelas e ventilação positiva.

Comparando o desempenho de lotes de frangos de corte criados em três sistemas, convencional (ventilação positiva com ventiladores), túnel (ventilação negativa com exaustores) e dark house (ventilação negativa com exaustores e controladores de luminosidade), Verdi (2009) verificou um melhor ganho de peso diário nos sistemas que utilizaram ventilação negativa, corroborando com os resultados encontrados no presente trabalho.

Os animais criados em aviários com sistema de ventilação negativa apresentaram maior valor por cabeça, no entanto, isso não se refletiu nos resultados de custo por cabeça e por quilo.

Em relação ao tipo de aviário, verificou-se menor conversão alimentar e maior IEP para os animais criados no sistema dark house quando comparado ao convencional (lona amarela), não havendo diferença em relação ao blue house $(p<$ $0,05)$ (Tabela 3). Estes resultados demonstram que os sistemas dark e blue house proporcionaram aos frangos um ambiente com melhor conforto, o que justifica os melhores resultados de desempenho em relação aos animais criados no sistema convencional.

Tabela 3 - Média \pm erro padrão das variáveis de desempenho e custo de frangos de corte em função do tipo de aviário (convencional/lona amarela, blue house e dark house)

\begin{tabular}{|c|c|c|c|c|}
\hline \multirow{2}{*}{ Variáveis } & \multicolumn{3}{|c|}{ Aviário } & \multirow{2}{*}{ Valor de $p$} \\
\hline & Lona amarela & Blue house & Dark house & \\
\hline Data de abate (dias) ${ }^{2}$ & $46,9 \pm 0,2$ & $47,3 \pm 0,3$ & $47,3 \pm 0,2$ & 0,467 \\
\hline Mortalidade $(\%)^{2 \star}$ & $3,5 \pm 0,3$ & $4,0 \pm 0,3$ & $3,9 \pm 0,4$ & 0,539 \\
\hline Conversão alimentar (kg/kg) & $1,797 \pm 0,0^{a}$ & $1,764 \pm 0,0^{b c}$ & $1,727 \pm 0,0^{c}$ & 0,000 \\
\hline Consumo médio $(\mathrm{kg})^{1}$ & $5,127 \pm 0,0$ & $5,091 \pm 0,0$ & $5,071 \pm 0,0$ & 0,849 \\
\hline Índice de eficiência produtiva (IEP) & $326,9 \pm 3,8^{c}$ & $332,8 \pm 4,4^{a b}$ & $345,9 \pm 5,0^{a}$ & 0,013 \\
\hline Ganho médio diário de peso $(\mathrm{g})^{1}$ & $60,7 \pm 0,4$ & $61,0 \pm 0,5$ & $62,0 \pm 0,5$ & 0,200 \\
\hline Peso médio $(\mathrm{kg})^{1}$ & $2,854 \pm 0,0$ & $2,890 \pm 0,0$ & $2,936 \pm 0,0$ & 0,132 \\
\hline Valor por cabeça (reais) & $0,48 \pm 0,0^{c}$ & $0,60 \pm 0,0^{a b}$ & $0,68 \pm 0,0^{a}$ & 0,000 \\
\hline Custo por cabeça (reais) ${ }^{1}$ & $5,8 \pm 0,1$ & $5,9 \pm 0,1$ & $5,9 \pm 0,1$ & 0,536 \\
\hline Custo por kg (reais) ${ }^{1}$ & $2,0 \pm 0,0$ & $2,0 \pm 0,0$ & $2,0 \pm 0,0$ & 0,886 \\
\hline
\end{tabular}

Nota: ${ }^{1}$ Não significativo pela análise de variância. ${ }^{2}$ Não significativo pelo teste Kruskal-Wallis. * Mortalidade do período de criação (média de 47 dias). Médias seguidas de letras diferentes na linha diferem pelo teste de Tukey HSD $(p<0,05)$. 
Avaliando o desempenho de frangos criados em três tipos diferentes de galpões (convencionais, blue house e dark house) no estado de São Paulo, Bichara (2009) observou melhor resultado de conversão alimentar para os animais criados no sistema dark house, mas o GMD não diferiu entre os três sistemas, corroborando com o resultado do presente trabalho.

Segundo Oliveira et al. (2014), quando se compara a conversão alimentar entre aviários convencionais e dark house, verifica-se conversão $13,51 \%$ melhor para o sistema dark house, no entanto, no presente trabalho o sistema dark house foi $3,89 \%$ melhor em comparação ao sistema lona amarela.

Gallo (2009) analisou dados de uma empresa brasileira oriundos de três tipos de galpões (convencional, ventilação negativa e dark house com luminosidade controlada) e salientou que dentre os sistemas comparados, a tecnologia dark house apresentou melhor conversão alimentar. Resultados semelhantes também foram encontrados por Rovaris et al. (2014) em relação à conversão alimentar em aviários dark house quando comparados aos aviários convencionais (ventilação positiva). Segundo o autor, aviários dark house permitem maior controle da ambiência no interior do aviário, oferecendo um ambiente mais confortável aos frangos alojados.

\section{Conclusão}

Conclui-se que animais da linhagem Hubbard e Cobb Slow apresentam melhores resultados de desempenho zootécnico (índice de eficiência produtiva, peso médio) do que animais da linhagem Cobb Fast.

Em relação ao sistema de ventilação (positiva e negativa) e tipos de aviário (dark house, blue house e convencional), melhores resultados de desempenho zootécnico são observados para sistemas que propiciam melhor ambiência, ou seja, ventilação negativa e com controle de luminosidade (dark e blue house). Por outro lado, em termos de custos, não houve diferenças nos parâmetros avaliados.

\section{Referências}

ABPA. Relatório Anual. 2020 [acesso 10 ago 2020]. Disponível em: https://abpa-br.org/relatorios/

Abreu VMN, Abreu PG. Os desafios da ambiência sobre os sistemas de aves no Brasil. R Bras Zootec. 2011;40(Supl Esp):1-14.

APAVI. Administração de granjas: índices zootécnicos. 2009 [acesso 01 jul 2012]. Disponível em: https://tinyurl.com/ y6zj5rr2

Api I, Takahashi SE, Mendes AS, Paixão SJ, Refati R, Restelatto R. Efeito da sexagem e linhagens sobre o desempenho e rendimento de carcaça de frangos de corte. Cienc Anim Bras. 2017;18:e32691.

Arruda JNT. Desempenho produtivo, rendimento de carcaça e bem-estar animal em frangos de corte de diferentes linhagens e densidades de alojamento [dissertação]. Dois Vizinhos: Universidade Tecnológica Federal do Paraná; 2013. 87 p.

Avicultura Industrial. Índice de Eficiência Produtiva de frangos atingem números recordes. 2018 [acesso $25 \mathrm{abr}$ 2018]. Disponível em: https://tinyurl.com/y2yac7w8

Avicultura Industrial. Sistema Blue House melhora produtividade em granjas. 2017 [acesso 10 ago 2020].

Disponível em: https://tinyurl.com/y3msjxyv

Bichara T. Aviário azul e dark-house para frangos de corte desenvolvendo novos conceitos para aviária pressão negativa. In: $10^{\circ}$ Simpósio Brasil Sul de Avicultura e $1^{\circ}$ Brasil Sul Poultry Fair; 31 mar - 2 abr 2009; Chapecó, SC. Chapecó: NUCLEOVET; 2009. p. 74-84.

Carvalho TMR, Moura DJ, Souza ZM, Souza GS, Bueno LGF. Qualidade da cama e do ar em diferentes condições de alojamento de frangos de corte. Pesq Agropec Bras. 2011;46(4):351-61.

Curi TMRC, Vercellino RA, Massari JM, Souza ZM, Moura DJ. Geoestatística para a avaliação do controle ambiental do sistema de ventilação em instalações comerciais para frangos de corte. Eng Agric. 2014;34(6):1062-74. 
Gallo BB. Dark House: Manejo x desempenho frente ao sistema tradicional. In: $10^{\circ}$ Simpósio Brasil Sul de Avicultura; 31 mar - 2 abr 2009; Chapecó, SC. Chapecó: NUCLEOVET; 2009.

Garcia Neto M, Campos EJ. Suscetibilidade de linhagens de frangos de corte à síndrome ascítica. Pesq Agropec Bras. 2004;39(8):803-8.

Lopes JCO. Técnico em agropecuária: Avicultura. Floriano, PI: EDUFPI; 2011.94 p.

Machado ST, Reis JGM, Vendrametto O, Nääs IA. Logística aplicada à produção de aves de corte: desafios no manejo pré-abate. Encicl Biosf. 2014;10(18):2108-22.

Moreira J, Mendes AA, Garcia EA, Oliveira RP, Garcia RG, Almeida ICL. Avaliação de desempenho, rendimento de carcaça e qualidade da carne do peito em frangos de linhagens de conformação versus convencionais. R Bras Zootec. 2003;32(6 Supl 1):1663-73.

Moro DN, Zanella I, Figueiredo EAP, Silva JHS. Desempenho produtivo de quatro linhagens de frangos de corte. Cienc Rural. 2005;35(2):446-9.

Oliveira KV, Cavichioli C, Andreazzi MA, Simonelli SM. Sistema dark house de produção de frangos de corte: uma revisão. In: $7^{\circ}$ Mostra Interna de Trabalhos de Iniciação Científica; 21-24 out 2014; Maringá, PR. Maringá: Cesumar; 2014. p. 1-5.

Pereira PC, Batista IA, Butolo EAF, Costa LL, Conde EM, Ruschel AS, et al. Avaliação do desempenho zootécnico e rendimento de carcaças de diferentes linhagens de frango de corte. Vet Not. 2019;25(2):161-71.
Revista Rural. Avicultura - criação de primeiro mundo. 2009 [acesso 10 ago 2020]. Disponível em: https://tinyurl.com/ y4ws6zc4

Rovaris E. Diferentes sistemas de alojamento de frangos de corte e de coleta de ovos em matrizeiros [dissertação]. Cuiabá: Universidade Federal de Mato Grosso; 2013. 60 p.

Rovaris E, Corrêa GSS, Corrêa AB, Caramori Jr JG, Luna UV, Assis SD. Desempenho de frangos de corte criados em aviários dark house versus convencional. Pubvet. 2014;8(18):Art.1778.

Rovaris E, Corrêa GSS, Corrêa BA, Luna UV. Avaliação da conversão alimentar e do ganho médio de peso diário de frangos de corte em dois sistemas de produção - dark house e convencional. In: 12o Congresso Brasileiro de Zootecnia; 13-18 mai 2012; Cuiabá, MT. Cuiabá: SBZ; 2012.

Silva IJO, Vieira FMC. Ambiência animal e as perdas produtivas no manejo pré-abate: o caso da avicultura de corte brasileira. Arch Zootec. 2010;59(R):113-31.

Souza CF, Baêta FC, Tinoco IFF, Freitas LCSR, Cândido MGL. Características ambientais dos aviários adotados atualmente no Brasil e respostas no desempenho produtivo. 2017 [acesso 10 ago 2020]. Disponível em: https://tinyurl. com/yxoromhm

Verdi P. Sistemas de automação em dark house para ambiência de frango de corte. In: Workshop Embrapa Suínos e Aves; 18-19 nov 2009; Concórdia, SC. Concórdia: EMBRAPA; 2009. p. 1-36. 